\title{
Variations
}

Variations

Revue internationale de théorie critique

$9 / 10 \mid 2007$

Les frontières de la politique

\section{La politique entre l'opératoire et le sentimental}

\section{Florent Jakob}

\section{(2) OpenEdition}

\section{Journals}

Édition électronique

URL : http://journals.openedition.org/variations/472

DOI : 10.4000/variations.472

ISSN : 1968-3960

\section{Éditeur}

Les amis de Variations

\section{Édition imprimée}

Date de publication : 1 juin 2007

Pagination : 79-89

\section{Référence électronique}

Florent Jakob, « La politique entre l'opératoire et le sentimental », Variations [En ligne], 9/10 | 2007, mis en ligne le 01 décembre 2012, consulté le 02 mai 2019. URL : http://journals.openedition.org/ variations/472; DOI : 10.4000/variations.472

Ce document a été généré automatiquement le 2 mai 2019.

Les ami•e•s de Variations 


\title{
La politique entre l'opératoire et le sentimental
}

\author{
Florent Jakob
}

1 Notre époque commande peut-être que nous pensions les «frontières du politique » à l'inverse du questionnement classique sur les limites qui devraient venir borner le politique, dans la mesure où le politique aurait par définition une tendance à s'étendre. Ce questionnement, renforcé pour longtemps par les phénomènes rassemblés sous le vaste concept de «totalitarisme ${ }^{1}$ ", engage classiquement un double point de vue. Pour freiner la capacité du politique à englober tout le social, on peut alors développer, d'un point de vue interne au politique, une théorie de la séparation ou de la répartition des pouvoirs, une théorie de la représentation politique, et/ou d'un point de vue externe, on peut alors s'appuyer sur des forces d'opposition au pouvoir, ou même extérieures au politique. Dans ce cadre, la société civile peut être l'exemple de ces forces qui viennent limiter l'extension du politique et en tracer les « frontières ». Cette société civile est ainsi caractérisée par le fait qu'elle n'appartient pas à la sphère du pouvoir et de ses fonctions (sphère qui ne définit évidemment pas entièrement le champ du politique, mais peut en être l'enjeu), bien qu'elle appartienne à la cité. Elle est alors aussi plus largement cet ensemble de personnes qui ne veut pas avoir à faire avec le politique, ne veut pas s'en mêler et ne s'occupe pas de tâches appartenant d'une manière ou d'une autre au fonctionnement politique (c'est-à-dire, le plus généralement et de la façon la plus minimale, au vivre ensemble). Tels sont les problèmes qui peuvent se poser si l'on estime que le politique est chose à limiter. Nous croyons au contraire que l'époque implique que nous pensions le problème de façon inverse. Autrement dit, loin de toute réflexion sur le totalitarisme (et la validité d'un tel concept reste encore à peser), il ne s'agira pas ici de s'enquérir des lieux que le politique ne doit pas investir, ni des moyens de le limiter.

\section{La réduction du politique à l'opératoire}

2 Au contraire, nous voudrions plutôt nous demander - et cela nous semble plus conforme à ce qui a lieu actuellement avec le politique - ce qui se joue de l'autre côté des frontières 
du politique. Qu'arrive-t-il au bord du politique pour que son territoire, ou le territoire qu'il pourrait revendiquer, soit de plus en plus étroit? Sous quelles formes se jouent les combats aux frontières du politique, combat dont l'enjeu n'est peut-être pas moindre que la mort - ou du moins l'extinction provisoire -, du politique? Sinon sa réduction à un ensemble de procédures, un mode opératoire, opposé à une action qui se refuserait à être politique, sinon même à être action, mais s'en tiendrait à l'assistance aux victimes et au langage de ce secours, un mode sentimental.

3 On pourrait essayer de rassembler sous quelques traits les expansions qui se font contre le politique et qui expliquent ou désignent ses rétrécissements. À l'encontre des frontières du politique, si celui-ci peut être classiquement entendu comme domaine public, expression d'une classe, d'un territoire... on notera :

1. la privatisation, le devenir privé, de l'espace politique comme espace public².

2. la prise en charge technicisée et professionnalisée du politique comme ce qui relevait du domaine public.

3. la «globalisation» ou «mondialisation» comme négation du territoire et de la politique comme expérience localisée.

4 Ces trois négations historiques - qui ne sont peut-être que d'intenses accélérations de ce que Marx préfigurait probablement dans le Manifeste communiste - du politique plus que jamais à l'œuvre rappellent que les «frontières » du politique sont aujourd'hui plus qu'enfoncées.

5 À constater ce rétrécissement du domaine politique, on pourrait croire que deux mouvements discontinus et parfaitement opposés ont été expérimentés en un siècle: celui visant à une totalisation politique et celui confinant à un anéantissement du politique. Derrière une opposition aussi massive, on retrouverait ce que l'on a nommé "totalitarisme» et ce que l'on nomme «libéralisme». La démocratie libérale, accompagnée dans son mouvement par les trois traits principaux dégagés ci-dessus, marquerait la sortie, l'épuration, la pacification d'un temps relevant de la guerre civile ${ }^{3}$. C'est certainement sur un temps plus long, et avec la " guerre civile européenne » comme arrière-fond qu'il faut penser une semblable évolution. Quoi qu'il en soit, cette épuration du politique serait marquée, de façon conjointe, objectivement par le retrait des prérogatives du gouvernement, et subjectivement par l'apolitisme ou la dépolitisation généralisés. Il apparaît problématique de séparer entièrement l'apolitisme contemporain et sa revendication dès qu'il prend les formes politiques du refus de l'organisation, de l'encadrement, du mouvement libéral d'ensemble qui attaque par définition toute structure, toute réglementation, toute institution en tant qu'elles sont des obstacles ${ }^{4}$. Mais ce n'est pas ce lien que nous cherchons à faire. Observons simplement que cohabitent temporellement deux phénomènes: un mode de réduction du politique à l'opératoire, et un refus de l'engagement, de l'action politique, une volonté de se tenir à l'écart, ou alors de s'engager sur des motivations relevant de l'humanitarisme.

6 Cette attaque de la structure par le libéralisme n'est rien de nouveau. On relira à ce titre avec intérêt les volontés qui s'expriment au moment même où le modèle libéral américain se constitue en défenseur de la liberté, c'est-à-dire à l'aube de la guerre froide. À ce moment qui configure et redistribue le monde contemporain, après la Seconde Guerre mondiale, et qui marque la fin de cette "guerre civile européenne ", juste avant de promulguer le plan Marshall, le 6 mars 1947, le président américain H. Truman affirme la volonté des Etats-Unis de se débarrasser des contrôles exercés sur le commerce par les 
gouvernements nationaux: "Le système commercial le moins favorable à la liberté d'entreprise est celui dans lequel ce sont les gouvernements qui décident ». Et il annonce la création prochaine de l'Organisation Mondiale du Commerce, qui « limitera l'actuelle liberté des gouvernements d'imposer à leur commerce international des réglementations administratives détaillées». Il s'agit alors «de limiter ces contrôles à des cas exceptionnels dans l'avenir immédiat et d'y renoncer entièrement aussitôt que cela sera possible ${ }^{5}$. Autrement dit, frontières terrestres, légales, publiques, gouvernementales et politiques doivent être ouvertes au capital industriel et financier américain ; les États et les gouvernements en tant que tels représentent des obstacles dont il s'agit d'amoindrir et de limiter la portée. Il est inutile de développer ce point davantage tant est visible à chacun le glissement ou l'abandon à la sphère privée d'anciennes prérogatives publiques. La réalité semble être pour l'instant que ce qu'il reste alors n'est plus qu'une gestion, une administration et de moins en moins de politique. Simple mode opératoire ${ }^{6}$.

7 À la crainte de voir la maison se confondre avec la cité, il nous faut donc maintenant vivre celle de la cité se confondant avec la maison. De surcroît, une maison elle-même principalement construite, remplie de productions entièrement privées, et dont seules les normes de cette production font l'objet d'un accord administratif (accord présenté alors comme politique, là où il ne s'agit que d'une régulation).

Mais dans le temps même où les gouvernements restreignent "délibérément » leur souveraineté et leur capacité d'action, on a pu et on peut continuer de voir la politique quitter le champ social et la "société civile ». Il n'y a peut-être pas là de rapport de causalité (du moins ne sommes-nous pas en mesure de le conceptualiser), mais il y a là en tout cas une indication à ne pas mener trop rapidement la critique du pouvoir et du gouvernement en tant que celle-ci mènerait à davantage de politique, comme à plus de liberté.

Et s'il y a une réaction légitime et fort compréhensible à cette réalité « totalitaire » dans le retrait hors du politique, dans la dépolitisation, dans la revendication apolitique de mouvements qui sont pourtant politiques par leur essence, il reste que ce n'est encore là qu'une réaction, et cette libération du politique par le renforcement constant de la sphère privée et la destruction de toute collectivité, voire de toute institution, est ce qui détermine aujourd'hui les frontières du politique. La question s'est donc historiquement inversée : elle n'est plus «à quoi ne s'étend pas le politique ? ", mais «à quelle extension peut encore prétendre le politique?».

\section{Passivité et action politique}

Jean-Luc Nancy observe :

Le témoignage le plus important et le plus pénible du monde moderne, celui qui rassemble peut-être tous les autres témoignages que cette époque se trouve chargée d'assumer, en vertu d'on ne sait quel décret ou de quelle nécessité [...], est le témoignage de la dissolution, de la dislocation ou de la conflagration de la communautér.

11 Pourquoi, si le politique disparaît de sa sphère traditionnelle et limitée, n'est-il pas soutenu et repris par ailleurs, mais au contraire délaissé et refusé, pourquoi nous refusons-nous à agir politiquement ? Et si tant est que nous le voulions, nous ne le faisons pas. La question des frontières du politique ne deviendrait-elle pas alors aujourd'hui celle des frontières de l'action ? Ni « que faire ? ", question retournée à l'attente, ni « que dois- 
je faire? » question morale sur laquelle les penseurs libéraux aimeraient rabattre la question politique, mais peut-être davantage « que puis-je faire?». Question qui n'est pas sans connoter une impuissance, et une position de réaction plutôt que d'action. La catégorie ou l'idée de l'action elle-même n'aurait-elle pas aujourd'hui été privatisée ?

À l'action, on aura toujours opposé la passivité. Il y a là continuité (fût-ce dans le renversement de valeur de l'une par rapport à l'autre entre le monde antique et le christianisme) : ce que je fais et ce qui m'arrive. Ce sur quoi ou avec quoi j'agis et ce que je contemple. Or, s'il y a vraisemblablement un tour de force achevé constitutif de l'idéologie présente c'est de rendre tout ce qui se fait réductible à ce qui m'arrive. Nous n'entendons pas ici fonder l'apolitisme contemporain, ni même l'expliquer. Nous nous contenterons de décrire dans les lignes suivantes une conjonction qui s'est faite et qui entrave au moins provisoirement toute action possible. Autrement dit, nous proposons ici quelques éléments de description de la réduction des agents à la passivité. Seconde réduction sans laquelle la première (du politique à l'opératoire) ne serait probablement pas possible, elle représente sa part subjective, et les deux se correspondent et sont historiquement solidaires.

Lukacs notait que la passivité était une résultante même de la production capitaliste et qu'on pouvait la déduire en suivant le fil des analyses du Capital.

L'homme n'apparaît ni objectivement, ni dans son comportement à l'égard du processus du travail comme le véritable porteur de ce processus, il est incorporé comme partie mécanisée dans un système mécanique qu'il trouve devant lui, achevé et fonctionnant dans une totale indépendance par rapport à lui, aux lois duquel il doit se soumettre. Cette soumission s'accroît encore du fait que plus la rationalisation et la mécanisation du processus du travail augmentent, plus l'activité du travailleur perd son caractère d'activité pour devenir une attitude contemplative ${ }^{8}$.

L'attitude contemplative se distingue et s'oppose en premier lieu à l'activité. Là où je n'agis pas, plus encore là où je n'agis plus, là où je me suis (ou j'ai été) dégagé de l'action, je peux contempler, il ne me reste qu'à contempler. Ce avec quoi j'avais rapport et auquel j'étais lié et uni, est maintenant objet pour moi.

15 En second lieu, l'attitude contemplative suppose encore le retrait de cette conscience de l'action possible. Je deviens contemplatif parce qu'en ce domaine l'action n'a plus de sens pour moi. C'est là le premier temps de la séparation. Si cette action ne représente plus une possibilité pour moi, si elle est maintenant entièrement en dehors de moi, alors je peux la contempler elle-même. Aussi, cette première séparation peut ensuite être spectacularisée, c'est-à-dire redoublée. Guy Debord, on le sait bien, thématise le spectacle et la spectacularisation. Et il le fait sur le fond de cette histoire que nous avons décrite, la guerre civile européenne s'éloigne, cet après-guerre aura été l'occasion d'une rare accumulation du capital, le politique se retire, et il perçoit déjà qu'il va être désinvesti. De manière significative, dans l'élaboration de sa critique du spectacle, Debord reprend une partie de la phrase de Lukacs que nous venons de mentionner. Nous retiendrons ici deux formules de La Société du spectacle. Sachant que le spectacle est « le Capital à un tel degré d'accumulation qu'il devient image » (\$34), il ne constitue pas pour autant « un ensemble d'images, mais un rapport social entre des personnes, médiatisé par des images.» Autrement dit, devenant image, et image de la marchandise, image de la vie sous le règne de la marchandise, la contemplation elle-même s'isole de moi, et je contemple la contemplation elle-même. En tant que rapport social médiatisé, aliéné et devenu invisible, l'image retrouve certes le caractère de la marchandise, mais aussi celui de la 
dépossession et de la médiatisation, supplémentaires et dédoublées. La spectralisation augmente. Là où le travailleur de Lukacs était fantôme de son activité au premier degré, l'homme qui vit à l'époque du spectacle constitué - nous - est passif au second degré. L'action s'est éloignée d'un cran supplémentaire. Et la société devient elle-même ce réseau spectral. Dépossession de l'action, spectacularisation de l'action, atomisation de celui qui pourrait agir, séparation de ceux qui devraient agir et d'eux-mêmes à l'égard de ce sur quoi ils devraient agir, toutes ces passivités sont mises en abîme et sériées ${ }^{9}$. Tels sont les traits de la situation sur le fond de laquelle le politique devrait étendre son territoire. Mais comment les dépossédés peuvent-ils donc faire de la politique, si faire de la politique c'est initier, entamer, commencer, porter le conflit, entrer en rapport à l'autre, bref se départir de la position contemplative et passive, et créer? Comment le dépossédé sort-il de sa dépossession?

\section{La violence comme problème}

La première réponse est certainement la violence. La violence fondatrice, comme la nomme Walter Benjamin, la violence constitutive, ou encore la violence simplement destructrice qui était déjà à l'œuvre dans les premières actions dirigées contre le monde de la production capitaliste, comme celle du mouvement chartiste en Angleterre. La violence comme irruption, le passage à l'acte, est sans doute le moyen de tout rapport, ainsi que le risque contenu dans tout rapport aux frontières du politique. Mais si la violence est toujours pour le dépossédé le moyen de faire irruption et la fin de la passivité, il est connu que ce moyen que je peux initier, mais dont je ne peux prévoir les effets, et les séries qu'il ouvre, n'est peut-être pas réductible à un simple instrument. Cette simple question de l'instrumentalisation de la violence dans l'histoire est aujourd'hui restée sans réponse, et surtout a purement disparu. La violence libérale reste seule, et ne passe bien évidemment pas pour telle. Dès lors, si la passivité détermine la situation présente, c'est que la violence ne veut pas être employée, c'est comme dirait Michel Foucault à l'égard de la révolution, on ne sait plus si elle est « désirable $»^{10}$ ? Qu'en est-il de la violence aujourd'hui ?

Travaillant la transformation de la question de la Terreur révolutionnaire, le problème de sa réception présente, l'historienne Sophie Wahnich note: «Ce qui s'est joué là, c'est la figure du mal historique, l'incapacité à régler pacifiquement des conflits politiques, c'està-dire sans violence faite au corps, sans donner la mort. Être un héritier heureux de la Révolution française, c'est devenir complice d'un crime historique. Ainsi s'efface le caractère de laboratoire politique de l'événement au profit d'une question morale $»^{11}$.

\section{La conjonction de l'impensé de la violence et de la passivité : le sentimental en lieu et place du politique}

De la politique vient donc le mal. Comprenons bien ici qu'il n'est nullement question de nier la violence historique, les tragédies qu'elle porte, mais qu'il s'agit de parvenir à une description de la configuration actuelle dans laquelle s'effondrent les frontières du politique si peu de temps après avoir semblé s'étendre indéfiniment. Mais à travers les passages de l'activité à la contemplation décrits ci-dessus, du problème de la violence thématisé ici et de l'histoire à son recouvrement par une morale et une "sagesse 
intemporelle » pour reprendre les mots de Traverso, et comme l'illustrait le travail de Wahnich sur la Révolution française, passages encore de la politique à la dépolitisation et à l'apolitisme, nous voudrions constater que l'impensé de la violence pèse significativement sur la génération qui devrait aujourd'hui agir. Il constitue une entrave qui redouble tout autant qu'il justifie après coup la passivité que nous avons précédemment décrite. Car si certes tout est fait pour que je ne me pose pas la question d'agir, ni même que je désire agir politiquement, en admettant que je veuille me départir de ma passivité et passer à l'action, cette donnée profonde et héritée comme le discours qui vient couvrir moralement cette absence historique de solution me retient de le faire. Il y a jonction de l'impensé de la violence et de la passivité. Cette jonction est certainement l'une des sources profondes de l'absence de relèves des frontières du politique par une quelconque partie de la dite « société civile ».

De la même manière, cette jonction permet d'éclairer le mode " positif » sur lequel se montre ce refus du politique: l'engagement tel qu'il a lieu aujourd'hui, associatif et surtout avant tout humanitaire.

En effet, si les frontières du politique semblent alors se refermer sur elles-mêmes, aspirées de l'intérieur, comme repoussées par l'extérieur, c'est que l'action politique se retrouve pressée entre l'opératoire gestionnaire d'une part, et la limite sentimentale. L'ancien champ politique se partage entre ces deux forces qui s'assurent et se solidifient réciproquement, assurant une grande force à l'ordre libéral. Dans le domaine de la gestion politique, on est " aux affaires ", et là serait le sérieux. On gère - c'est-à-dire on continue, on perpétue ${ }^{12}$, mais on ne commence plus, on n'initie plus, on ne propose plus de visées lointaines (et il n'est pas la peine de rappeler ici à quel point ce lexique de la gestion a envahi toute la langue courante, et jusqu'aux comportements et propos de tout un chacun. Même dans sa vie, on est "aux affaires"). Tout ce qui ne ressortit pas à ce cercle de l'action conforme à l'ordre que désigne la " gestion » est à l'inverse disqualifié comme action, et doit être reconduit à telle ou telle frustration, à telle motivation nonpolitique - dont on essaiera de pointer qu'elle est encore imaginaire plutôt que réelle ${ }^{13}$. L'action s'annule par sa motivation. La motivation étant d'ordre psychologique et, au mieux, d'ordre sociologique, toute action n'est alors que réaction.

On comprendra pourquoi l'engagement politique n'a ici plus guère d'espace ni de sens. Il convient alors de s'engager sur le terrain qui est proprement celui de l'engagement, et qui n'est pas une action politique, terrain qui devient alors bien celui du sentiment, et dont le langage est bien celui du cœur, de même que son nom porte en lui l'absence de toute dimension conflictuelle : « l'humanitaire».

En effet, on ne s'engage presque plus en politique ${ }^{14}$, on se désengage plutôt, alors qu'on s'engage dans l'humanitaire. La "noblesse» de la cause humanitaire qui se situe en dehors des frontières du politique et participe certainement de sa décomposition ne vient pas d'ailleurs. L'importance commémorative, médiatique, paradigmatique pour notre époque, de la victime a été déjà fréquemment relevée. La victime est celle du politique : elle ne lui a rien demandé et s'y trouve mêlée, elle est au-dehors de ses frontières au sens où elle n'est pas nécessairement acteur, mais se trouve être malgré elle, ou sans l'avoir voulu là même où s'affrontent les forces, parce que le politique tendrait toujours à déborder. On pâtit du politique, voilà pourquoi les effets doivent en être diminués, il est l'objet d'une menace. 

s'approprier la violence historique, ou une réflexion sur cette violence, se tourne vers la victime, et que la « compassion » devienne alors le motif le plus récurrent, le plus louable de l'action. On s'engage hors du politique, à partir de déterminations affectives, qui se revendiquent comme telles, et pour ceux qui en sont victimes. L'engagement devient prioritairement le fait de l'humanitaire. L'action sera toujours plus légitime dans le champ (apolitique, et faut-il dire anti-politique ?) de l'humanitaire. En effet, ce qui peut alors librement triompher, c'est la compassion. L'humanitaire est permis et valorisé car il s'opère au nom même de valeurs passives. Et à mesure que le politique s'est retiré, on aura vu croître la sphère de l'humanitaire. À ce titre, l'histoire de l'humanitaire et de ses fondateurs se joue bien dans cette démarcation à l'égard du politique, de son terrain, et de ses motivations. trouve objectivement l'individu et l'absence d'une réponse à cette question de la violence empêchent subjectivement toute autre attitude d'engagement. Pendant le temps qu'elles favorisent tous les engagements qui se situent hors des frontières du politique (l'engagement humanitaire, le fait de se consacrer par principe à la victime, qui est en général la victime - médiate ou immédiate - du politique). Le problème est donc celui d'une «critique de la violence » pour reprendre ici un titre célèbre. La violence, comme violence conflictuelle, reste impensée une fois de plus, et peut-être sous une forme encore jamais atteinte dans son raffinement, c'est la morale qui vient la recouvrir et l'obstruer. Là même où on prétendrait la morale dépassée, elle vient remplacer l'histoire, et la question de la violence reste en suspens. Son suspens est tel qu'elle ne nous apparaît plus même comme une question digne d'importance. Mais si tel était véritablement le cas, qu'est-ce qui obstinément nous repousse à l'idée de nous engager, c'est-à-dire de nous introduire à l'intérieur des frontières du politique pour les assumer, introduction qui implique la sortie de notre contemplation et la volonté de s'émanciper de cette séparation généralisée? suffisamment puissant pour réduire l'homme à la contemplation, et d'autre part, le contemplatif est devenu trop incertain sur les possibilités de son action (c'est-à-dire sur la violence qui initie et que son action ne peut pas ne pas impliquer) pour vouloir qu'il en aille autrement. Inscrire la pensée et la volonté politique dans une passivité : on est symptôme toujours, on n'est jamais créateur. Déposséder celui qui pourrait être acteur, créateur, c'est le réduire à l'esclavage. La conjonction de la dépossession comme image (spectacularisation) et de la défaite devenue incapacité de savoir comment agir et reposer le problème de l'action, tel est peut-être l'horizon à partir duquel nous devrions aujourd'hui poser les problèmes politiques.

À un tel carrefour, qui ne consiste pas à théoriser l'impuissance intellectuelle, les frontières du politique ne peuvent pour l'instant que continuer de fondre. Le caractère moral et technique, sentimental et opératoire des trois camps présents à gauche n'en donne-t-il pas la preuve ? Purisme moral et inaction de la gauche "révolutionnaire », technicité affichée et sentimentalisme du discours de la gauche « libérale », entre les deux 
le signe et le symptôme que toute reconfiguration reste particulièrement difficile et inaboutie. La seule critique des partis comme modèle dépassé, pas davantage que l'attachement à cette forme, ne peut actuellement rendre compte d'une telle situation, ni ne suffit à le faire si ne sont pas davantage explicités les problèmes hérités.

\section{NOTES}

1. Pour une vue d'ensemble, cf. E. Traverso, Le Totalitarisme, Le Seuil, 2001.

2. Il y a un quart de siècle, Ariès travaillant sur l'évolution de la famille concluait déjà : " Au cours $\mathrm{du}$ XXe siècle, et plus particulièrement depuis la Seconde Guerre mondiale et la crise de prospérité qui l'a suivie, l'équilibre a été rompu, l'espace public a été refoulé et a presque disparu, sauf dans les pays méditerranéens, et l'espace privé a envahi toute la société comme un cancer. La ville a enregistré cette évolution... » («La famille », P. Ariès, in Philosopher, Fayard, 1980, p. 227). Avant lui, H. Arendt note à propos des Français et du «petit bonheur»: "Cet épanouissement du privé, cet enchantement, dirait-on, de tout un peuple ne fait pas un domaine public, mais au contraire signifie seulement que le domaine public s'est presque entièrement résorbé... » (Condition de l'homme moderne, Calmann-Lévy, 1983, p. 92)

3. Cette guerre civile européenne n'est évidemment pas ici une reprise du concept et des analyses de Nolte. On se référera à l'emploi qu'en fait $\mathrm{E}$. Traverso dans son dernier ouvrage, A feu et à sang.

4. À ce titre, il reste problématique d'opérer une critique des partis comme instrument politique, sans avoir déterminé au préalable la position depuis laquelle on opère cette critique. N'appartient-elle pas à une conscience profondément déterminée par ce mouvement de la société bourgeoise tel que le définit Marx? Mais que faire si les partis en tant que tels sont eux-mêmes devenus des obstacles? Là n'est pas l'enjeu de cet article.

5. « Pourquoi le plan Marshall ? », cité par J-J. Marie, Staline, Fayard, 2001, p. 759.

6. S'il se peut qu'elle soit elle-même - peu importe ici sa réception - partie prenante dans ce dégagement du politique à l'égard du social pensé comme conflit, si elle représente peut-être un maillon dans cette chaîne qui a constitué le conflit en interdit et poussé à sa dérivation sociologisante et psychologisante, c'est-à-dire non politique, Arendt décrit bien la situation de ce problème: "Toute victoire complète de la société produit une sorte de "fiction communiste" dont la première caractéristique politique est bien, en effet, le gouvernement de la "main invisible", autrement dit celui de l'anonymat. Ce que nous appelons traditionnellement État et gouvernement fait place à l'administration pure - situation que Marx prédit correctement sous le nom de "dépérissement de l'État", en commettant toutefois l'erreur de supposer que seule la révolution peut la provoquer, et plus encore, de croire que ce triomphe de la société entraînerait éventuellement l'apparition du "règne de la liberté" " in Condition de l'homme moderne, ibid. p. 84.

7. J.-L. Nancy, La communauté désœuvrée, C. Bourgois, 1990.

8. Cf. G. Lukacs, Histoire et conscience de classe, Minuit, 1960 pp. 116-117.

9. À titre purement indicatif, et parmi mille autres aspects possibles. La dépossession est mise en abîme et en série par exemple par ce que R. Castel analyse - peu importe ici les désaccords éventuels quant à son rapport critique à Freud - comme «Psychanalysme», et la psychologisation généralisée de la société, ce qui contribue à son atomisation infinie. Ce n'est plus mon action qui importe, mais c'est l'écoute de mes motifs dans lesquels elle s'ancre. Un exemple trivial, mais parlant de ce phénomène peut se lire dans les «portraits " du journal 
Libération, dont on connaît et l'histoire et l'évolution. Ces portraits consistent fréquemment par la biographie ou la confidence rapportée à ramener l'homme politique, le militant, ou le penseur à une histoire personnelle, et aux déterminations affectives de son parcours. Autrement dit, les motifs de l'action ne peuvent être "sérieusement» politiques ou sociaux, mais ils sont individués, et appartiennent à mon histoire. Cf. in Philosopher, ibid. et sur Robert Castel, M. Foucault, Dits et Écrits, III. Gallimard, 1994. Comme motif plus nettement répressif et dissociant à l'intérieur du social, on observera par exemple le traitement individuel du chômage. Comme autres traits de cette position de la contemplation, et de la position de répression qu'elle pose comme entrave à l'action, à la création, on constatera l'omniprésence du commentaire dans les sciences sociales, le second degré et la référentialité, la «citation » dans les arts, ou encore la bascule, qu'expliquait par exemple Genette au sein de la littérature vers son pôle critique (cf. Figures II, Seuil, 1969).

10. À titre de simple remarque, on peut conjecturer que la fascination qu'exerce et la médiatisation que rencontre aujourd'hui par exemple la figure profondément anti-politique et anti-sociale du tueur en série, celui qui passe à l'acte dans le domaine entièrement privé, est certainement l'expression de cette dépossession. Seuls ceux que l'on a entièrement soustraits à l'action, à la simple possibilité d'agir, seuls les dépossédés peuvent projeter négativement leur impuissance pour donner de l'importance à ce type de figure.

11. La liberté ou la mort, La Fabrique, 2003, p. 15.

12. Ce qui n'implique en rien le recours à une tradition qui fasse autorité et qui par là serait légitime. Au contraire l'amnésie historique (dont le devoir de mémoire n'est peut-être pas l'opposé) peut être le garant d'une telle gestion. Car la mémoire et la tradition sont aussi toujours les sources et les appuis pour une contestation. Si l'histoire disparaît - et même de la culture des « élites " - c'est un critère d'opposition écartée. Muséale, l'histoire est alors débarrassée du conflit, et l'on peut revendiquer toute appartenance, puisque ces appartenances n'engagent plus aucune prise de parti. Celles qui ne sont pas susceptibles - ou pas encore susceptibles - d'une telle muséification disparaissent simplement.

13. Ainsi, dans son essai qui fit date, François Furet reconduit l'action révolutionnaire au langage des passions. Le premier chapitre traitant de la genèse se nomme «La passion révolutionnaire ", il y est question tout de suite de "magie », et le chapitre qui suit la Première Guerre mondiale s'intitule «Le charme universel d'octobre ». Et la puissance d'Octobre s'exerce justement sur les « imaginations». Arendt notait à la suite de la phrase sur les français citée ci-dessus que justement le charme n'était pas politique, et que la politique se faisait sur le refus du charme. L'opération menée par Furet devient alors évidente. Cf. Le passé d'une illusion, essai sur l'idée communiste au XXe siècle, Robert Laffont/Calmann-Lévy, 1995.

14. Au sens où cet engagement se lierait à l'émancipation, bien entendu. Nous ne nions pas ici l'inscription quantitative de personnes que revendiquaient récemment par exemple le «PS » ou l'«UMP».

15. Il est remarquable que même l'extrême gauche, et dans ses versions apparemment les plus dures, les plus issues du « socialisme scientifique » fait justement appel à ce langage du cœur, de la « vie » en tant que telle. 
AUTEUR

FLORENT JAKOB

Agrégé de philosophie 